\title{
The experimental design method of impact dynamics for vehicle collision
}

\author{
Zuo Baogui
}

\author{
Automotive Engineering institute, Jiangxi University of Technology, Nanchang 330098, China
}

Key words: Vehicle collision; Impact mechanics; Experimental design; Experiment teaching

\begin{abstract}
Theoretical Mechanics is the science to study the universal law of the object's mechanical motion. Proceeding from the few most essential laws, it applies the methods of logical reasoning and mathematical deduction so as to obtain a series of theorem, law and formula which reveals the principles of mechanical motions and to build a precise and complete theoretical system. Experiments in mechanics can illustrate the abstract mechanical behavior, contributing to students' understanding of the mechanical concepts. With the development of infrastructure construction in institutions of higher education and mechanical subjects, it is ready for colleges to open mechanical experimental courses with higher level and more contents. According to the principles and methods of kinetic parameter tests, in the article, an experimental method is designed to simulate initially the typical impact experiment in vehicle engineering.
\end{abstract}

\section{Introduction}

As the British scientist Bacon said, "No experiment, no science". Professor Ding Zaozhong who explored the nature through longtime experiments and has achieved great success once said, "Doing experiments is very important, because no theory works without being testified by experiments. Experiments can overturn theories while the reverse situation can never happen."

Since 1950s, the higher engineering education in China has been imitating the Soviet mode ---- to open theoretical mechanics courses for many majors. The syllabus of such a course emphasizes theoretical lectures without any experiment. As an important elementary course for engineering technology, theoretical mechanics involves abundant concepts, axioms, principles, theorems and mechanical models. Many mechanical laws do exist but they are not intuitive. It is difficult for pure lectures with theoretical and abstract contents to achieve a satisfactory teaching effect. For a long time, students state that theoretical mechanics is too profound to be understood, which is harder to master compared with mechanics of materials.

Mechanical experiments can demonstrate the abstract mechanical behavior vividly, contributing to students' understanding of the concept. With the development of infrastructure construction in institutions of higher education and mechanical subjects, it is ready for colleges to open mechanical experimental courses with higher level and more contents. Thus, the National Engineering Mechanics Course Steering Committee demands that the qualified institutions should reform their teaching to make sure the experiments for theoretical mechanics course can reach 15-20 hours. The Ministry of Education is also organizing the experts in China's key universities to formulate the essential requirements for the basic mechanical experiments teaching and compile the textbooks. Such an action helps to promote the teaching experience of mechanical experiment courses and formulate the experiment projects (modules), essential requirements and criteria so as to universalize and standardize the basic mechanical experiments gradually. The textbooks can be designed as 
representative, universal and selective modules, enabling more colleges and universities to open the experiment courses and choose proper textbooks, according to their own condition.

There are various impact experimental devices. In the form, they are horizontal, vertical, tilting .etc. In terms of the impact models, they are freefall, drop hammer, horizontal impacted, drum falling, tilting impacted, pendulum bob, vibratory, impulse, catapulting .etc. Horizontal and catapulting impact experimental devices are generally applied to the vehicle collision experiment. Falling or dropping-style devices are utilized in packaging or to test the shock resistance of some products (eg. helmets). Freefall-style, drum falling-style and tilting-style devices are mainly used in packaging. Particularly, it is flexible for the tilting-style devices to adjust the speed and convenient to drag, while pendulum bob-style ones are adopted to test the material's impact strength. And vibratory and impulse-style devices are applied to inspect the lifetime of electronic products or impact strength.

In this essay, through the analysis and research of the existing models, an impact dynamics experiment model and teaching method that are suitable for vehicle engineering is designed.

\section{The experimental design method}

The design of impact models is different from that of other vehicle engineering. Here are some aspects to be considered.

(1) There must be stroke length and track width on the test bed to fulfill the demand of different working conditions and testing objects.

(2) There must be a dip angle for the track and it can be adjusted. If necessary, a rubber string assistance device must be added to meet the need of high-speed impact experiments.

(3) The stiffness coefficient of collision barrier must be adjustable.

(4) The impact experimental trolley must be universal with enough strength and stiffness.

(5) Under the premise of reliable data, parameters test must be convenient and swift so that it is likely to collect signals and intelligentize the analysis.

(6) Combine the pleasant appearance with the ergonomic operation.

There is a rubber hook housing to install the catapulting string both in the front-end of the trolley and under the barrier. To make the adjustment of catapulting more convenient, the system has 11 hook holes which are meant to adjust impact speed or energy by changing the number of assistance strings.

With the development of electronic technology, especially the micro-electronic technique and electron devices, the tester tends to be designed to be digital, intelligent and modularized. When designing modern instruments, more and more experts advocate the concept of "device solution" which refers to a solution of adopting "characteristic" devices as much as possible during the circuit design, such as ASIC (application-specific integrated circuit), programmable integrated circuit, etc. Highly integrated chips should be utilized to simplify circuit and its design, thus improving the device's overall electric performance and making it more manufacturable and reliable with less expense.

The main program is mainly to initialize the system and display the update of the buffer. The system initialization contains parallel interface, timer, interruption, serial port, etc. When the contents to be displayed change, the update program in the display buffer will update the display.

1. Set variable s:s=1,2,3,4, as pz6,p15,pr4,p13 respectively and the others are 1 . For example, $\mathrm{Pl}=\mathrm{oxbf}$, if $\mathrm{s}$ is the month. It will judge whether any button has been pressed. The function related to 
the buttons will be carried out. And then it will be sent to the display circuit and the data to be displayed to the buffer.

2. The keys' functions. Set variables as FL) NCTION and f,f (1-10) which corresponds to different functions. D household with a people, and set variable $\mathrm{d}, \mathrm{d}$ with different values corresponding to different display models. When FUNCTION is to test the accelerated speed, implement the external interruption, enabling to respond to external interruption produced by the two photo-amplifiers (Fig .3-12).

3. The program's process of the display. Dynamic display. One update per $2 \mathrm{~ms}$. Choose three different LED or luminous diodes. Set display buffer. In the main program, the buffer should be updated in time (Send the data to be displayed, which are given by each key's function to the buffer). Every $2 \mathrm{~ms}$, the timer will break off, and data in the buffer will be sent through the serial port to be displayed.

4. Process the 5 sets of data. Set variable m (1-5) to indicate each group of accelerated speed display data. After the display, $m$ will add 1 automatically. When $m=5$, then it will return to 0 .

Design relevant parameters

$$
\bar{v}=\lim _{\Delta t \rightarrow 0} \frac{\Delta \bar{r}}{\Delta t}=\frac{d \tilde{r}}{d t}=\tilde{r}
$$

The speed here refers to the instantaneous speed of the moving point at a certain moment. The real instantaneous speed cannot be measured directly. But in the engineering measurement where the speed doesn't change a lot, comparing the average speed of moving points within a short time can approximate the instantaneous. In the article, advanced photoelectric technology is adopted in the velocity-measuring system. A specific light barrier is installed on the moving experimental trolley. Two light barrier bars are set in the distance of $10 \mathrm{~mm}$. A photo-amplifier is installed at the place where speed should be tested on the fixed bevel track. By measuring the interval between the two light barrier bars going through the photo-amplifier, the speed at which the trolley passes the point can be achieved. Because there are only $10 \mathrm{~mm}$ between the two bars, the speed that has been achieved can be seen as the instantaneous speed.

The engineering adopts the method of approximation to measure the instantaneous speed. That is, the average speed within a short time is regarded as the instantaneous speed for a certain moment.

If the photo-amplifier is set in the front, it will cause decelerating after the trolley's collision. The error is unacceptable. What's worse, it won't enable to complete the measurement. Conversely, if the photo-amplifier is set in the back, the achieved speed will not be the real collision speed but the speed of a certain moment before the collision, which also brings about error. But the error is relatively smaller. In conclusion, when we measure the collision speed, we should adjust the installing position of the photo-amplifier repeatedly to get a more accurate collision speed. The photo-amplifier is fixed with magnetic gauge stand will bring great convenience in the process of adjustment.

The speed measured by the light barrier consisting of two bars actually is the average speed between the two bars going through the photo-amplifier. We will approximate the average speed as the one of one bar passing the photo-amplifier. When the distance between the two bars is longor the moving point's accelerated speed is high, the achieved speed will show more systematic errors. 
Here is the principle of "double light barrier method". If the distance between the bars is d, the time of the first bar passing the photo-amplifier is $t 1$, the time for the second one is $t 2$, and the interval is $\mathrm{t} 12$, then we can use the following formula to get accelerated speed. $a=\left(\frac{d}{t 2}-\frac{d}{t 1}\right) / t 12$

Rolling friction is the most common phenomenon in the engineering. In order to improve the efficiency, lessen the labor intensity, rolling of the object usually replaces sliding. Dating back to Shang Dynasty, people in China used carriages for transportation. When heavy objects are to be carried, pipes will be laid under the objects, which makes a practical example. Although rolling friction is smaller than sliding, it is still a complicated phenomenon depending on numerous factors such as the distorted micro-slippage, elastic hysteresis, plastic deformation and adherence, etc.

\section{Vehicle collision experiment}

Throughout the world, about 1.2 million people are killed by traffic accidents every year, and over 30 million are injured. China is one of the countries where accidents happen frequently. In 2001, there were over 760,000 road accidents where 109,000 people died, 549,000 were injured and direct economic loss reached RMB3,05 billion. In 2002, about 770,000 traffic accidents happened with 109,000 deaths and 562,000 injured. The economic loss was RMB3,32 billion. Those casualties were most caused by vehicle collision. In the 1990s, the regulation ECE R94 and standard J208 that the Europe issued were based on FMVSS208. In China the existing CMVDR294 "About the design rules for protecting passengers in head-on collision" is the equivalent of the European ECE94. There are two kinds of difference. One, the collision angle in Europe changes from 30 degree to 0 degree. Two, because the figures of the Asian and European are different, " $\mathrm{H}$ " of the seat adjustment changes a bit.

To ensure the quality of vehicles, may countries make compulsory requirements for the safety of car collision through regulations, such as FMVSS in the USA, ECE/EEC in Europe. So does our country. The performance of the components related to safety has been regulated. Vehicle collision experiments are crucial in those regulations. The authority of those experimental data is undeniable when the safety of a vehicle is testified.

Since 1992, China has conducted the research of car's passive safety. Chinese Automotive Technology \& Research Centre, Automobile Research Institute in Tsinghua University, National Automobile Quality Supervision Test Centre (Xiangfan) all constructed pulley-simulation collision test bed. Afterwards, Tsinghua University began to experiment real vehicle collision on the rubber catapult-style test bed. The National Automobile Quality Supervision Test Centre both in Changchun and Xiangfan followed suit. The testing ground in Tongxian County of the Ministry of Transport also built similar device supported by continuous current dynamo. The collision system built by Chinese Automotive Technology \& Research Centre in 1999 has reached the word standard.

Vehicle collision experiment can be designed as follows.

Fix a seat on the trolley. Install a sensor respectively on both the back of the trolley and the seat. According to the requirement of the system, connect the wires, adjust the relevant parameters, and set the calibration of instrument. Then, pull the trolley upward to the preset height, set it free, and complete a collision experiment. The speed before the collision is measured and displayed by the system. After the accelerated speed is magnified and filtered and the sample is collected through sensors, the engineer analysis software will analyze and display it on the screen of the computer. 


\section{Acknowledgements}

This work was financially supported by the key subject building project (vehicle engineering) of Jiangxi University of Technology.

\section{References}

[1] Huisingh C, McGwin G, Wood J, et al. The driving visual field and a history of motor vehicle collision involvement in older drivers: a population-based examination[J]. Investigative ophthalmology \& visual science, 2015, 56(1): 132-138.

[2] Lewis G C, Platts-Mills T F, Liberzon I, et al. Incidence and predictors of acute psychological distress and dissociation after motor vehicle collision: a cross-sectional study[J]. Journal of Trauma \& Dissociation, 2014, 15(5): 527-547.

[3] Hunold K M, Sochor M R, McLean S A, et al. Ambulance transport rates after motor vehicle collision for older vs. younger adults: A population-based study[J]. Accident Analysis \& Prevention, 2014, 73: 373-379.

[4] Huisingh C, McGwin G, Orman K A, et al. Frequent falling and motor vehicle collision involvement of older drivers[J]. Journal of the American Geriatrics Society, 2014, 62(1): 123-129.

[5] Bolton J M, Au W, Walld R, et al. Parental bereavement after the death of an offspring in a motor vehicle collision: a population-based study[J]. American journal of epidemiology, 2014, 179(2): 177-185.

[6] $\mathrm{Hu} \mathrm{J}$, Bollen K, Lane S, et al. (100) Evaluation of the influence of stress-induced pain vulnerability and sociodemographic characteristics on acute pain after a motor vehicle collision using structural equation modeling[J]. The Journal of Pain, 2015, 16(4): S1.

[7] Wang X, Xie H, Cotton A S, et al. Early cortical thickness changes after mild traumatic brain injury following motor vehicle collision[J]. Journal of neurotrauma, 2014 (ja).

[8] Joerer S, Segata M, Bloessl B, et al. A vehicular networking perspective on estimating vehicle collision probability at intersections[J]. Vehicular Technology, IEEE Transactions on, 2014, 63(4): 1802-1812.

[9] Liu D, Solomon D, Hardy L. Investigating injury occurrence in motor vehicle collision using artificial neural networks[J]. Human Factors and Ergonomics in Manufacturing \& Service Industries, 2014.

[10]Wu C, Peng L, Huang Z, et al. A method of vehicle motion prediction and collision risk assessment with a simulated vehicular cyber physical system[J]. Transportation Research Part C: Emerging Technologies, 2014, 47: 179-191. 